\title{
Perfil microbiológico e alterações citológicas associadas as amostras cérvico-vaginais coletadas em uma instituição filantrópica no estado de Sergipe
}

Microbiological profile and cytological alterations associated with cervico-vaginal samples collected at a philanthropic institution in the state of Sergipe

Perfil microbiológico y alteraciones citológicas asociadas a muestras cérvico vaginales recolectadas en una institución filantrópica del estado de Sergipe

\section{Resumo}

O câncer de colo uterino é um problema de saúde pública, sendo o terceiro tipo de câncer mais comum em mulheres no Brasil, podendo ser diagnosticado através do exame citopatológico. O objetivo do trabalho é analisar o perfil microbiológico e alterações citológicas associadas as amostras cérvico-vaginais coletadas em uma instituição filantrópica, no estado de Sergipe. Trata-se de uma pesquisa retrospectiva, descritiva e transversal, em que foram analisados os resultados dos prontuários de mulheres que realizaram o exame citopátológico entre os meses de julho à outubro de 2017. No período de estudo, foram realizados 500 exames citopatológicos, sendo que 96\% (480/500) das amostras foram consideradas satisfatórias e 4\% (20/500) insatisfatórias. Ao analisar o perfil microbiológico, notou-se: Microbiota Mista em 31,2\% (156/500) dos casos, Cocos em 29,45 (147/500), Lactobacilos sem citólise em 16\% (80/500), Lactobacilos com citólise em 9,2\% (46/500), Bacilos supracitoplasmáticos em 7,4\% (37/500), Cocobacilos em 1,6\% (8/500), Cândida spp. em 2,4\% (12/500), Trichomonas vaginalis em 0,8\% (4/500) e ausência de microorganismos em 1,6\%. Já os achados citológicos foram representados da seguinte forma: Inflamação em 73,6\% (368/500) dos casos, Dupla Alteração, ou seja, Atrofia com Inflamação e Metaplasia Escamosa Imatura e/ou Inflamação com Metaplasia Escamosa imatura 13,4\% (64/500) dos casos, Atrofia com Inflamação em 9,4\% (47/500), dentro dos limites da Normalidade em 3,4\% (17/500) e Metaplasia Escamosa Imatura em 1\% (5/500) dos resultados. Portanto, o número de microorganismos encontrados nos resultados foram elevados. Em relação as alterações citológicas, foi observado que a inflamação estava presente na maioria dos casos.

Palavras-chave: Câncer de colo uterino; Microbiologia; Teste de Papanicolau.

\begin{abstract}
Cervical cancer is a public health problem, being the third most common type of cancer in women in Brazil, and it can be diagnosed through cytopathological examination. The objective of the work is to analyze the microbiological profile and cytological changes associated with cervicovaginal collected in a philanthropic institution, in the state of Sergipe. This is a retrospective, descriptive and cross-sectional research, in which the results of the medical records of women who underwent cytopathological examination between the months of July and October 2017 were carried out. During the study period, 500 cytopathological examinations were performed. 96\% (480/500) of the Subsidiaries were considered satisfactory and 4\% (20/500) unsatisfactory. When analyzing the microbiological profile, it is noted:
\end{abstract}


Mixed Microbiota in 31.2\% (156/500) of cases, Cocos in 29.45 (147/500), Lactobacilli without cytolysis in 16\% (80/500), Lactobacilli with cytolysis in 9.2\% (46/500), Supracytoplasmic bacilli in 7.4\% (37/500), Cocobacilli in $1.6 \%$ (8/500), Candida spp. in 2.4\% (12/500), Trichomonas vaginalis in 0.8\% (4/500) and Absence of microorganisms in $1.6 \%$. The cytological findings were represented as follows: Inflammation in $73.6 \%$ (368/500) of cases, Double Alteration, that is, Atrophy with Inflammation and Immature Squamous Metaplasia and/or Inflammation with Immature Squamous Metaplasia 13.4\% (64/500) of the cases, Atrophy with Inflammation in 9.4\% (47/500), within the limits of Normality in 3.4\% (17/500) and Immature Squamous Metaplasia in 1\% (5/500) of the results. Therefore, the number of microorganisms found in the results were high. Regarding cytological changes, it was observed that inflammation was present in most cases.

Keywords: Cervical cancer; Microbiology; Pap test.

\section{Resumen}

El cáncer del colo del útero es un problema de salud pública, siendo el tercer tipo de cáncer más común en mujeres en Brasil, y puede ser diagnosticado mediante examen citopatológico. El objetivo del trabajo y análisis del perfil microbiológico y alteraciones citológicas asociadas a la evidencia cervicovaginal recolectada en una institución filantrópica, no en el estado de Sergipe. Se trata de un estudio retrospectivo, descriptivo y transversal, en el que se analizaron los resultados de dos historias clínicas de mujeres que se sometieron a Papanicolaou entre julio y octubre de 2017. Durante el período de estudio se realizaron 500 pruebas de Papanicolaou, 96 de las cuales\% (480/500) de las muestras consideradas satisfactorias y 4\% (20/500) insatisfactorias. Analizar perfil microbiológico, nota: Microbiota mixta en 31,2\% (156/500) dos casos, Cocos en 29,45 (147/500), Lactobacilos sin citólisis en 16\% (80/500), Lactobacilos con citólisis en 9,2\% (46/500), bacilos supracitoplasmáticos en el 7,4\% (37/500), cocobacilos en el 1,6\% (8/500), Candida spp. en el 2,4\% (12/500), Trichomonas vaginalis en el 0,8\% (4/500) y ausencia de microorganismos en el 1,6\%. El dolor citológico se representa de la siguiente manera: inflamación en 73,6\% (368/500) dos casos, alteraciones dobles, es decir, atrofia con inflamación y metaplasia escamosa inmadura y / o inflamación con imagen de metaplasia escamosa 13,4\% (64/500) dos casos , Atrofia con inflamación en 9.4\% (47/500), dentro de dos límites de normalidad en $3.4 \%$ (17/500) y metaplasia escamosa inmadura en $1 \%$ (5/500) do resultados. Por tanto, la cantidad de microorganismos encontrados, obtuvimos resultados elevados. En cuanto a las alteraciones citológicas, se observó que la inflamación estuvo presente en más de dos casos.

Palabras clave: Cáncer de cuello uterino; Microbiología; Prueba de Papanicolaou.

\section{Introdução}

O câncer é um importante problema de saúde pública em países desenvolvidos e em desenvolvimento, contemplando um grupo de mais de cem enfermidades com características próprias. Esta patologia exibe mecanismo de proliferação celular desordenado, que não é controlado pelo organismo humano e que tem a capacidade de invadir outros órgãos e tecidos (Taquary et al. 2018).

Entre diversos tipos de câncer, o câncer de colo uterino (CCU) anualmente apresenta a incidência de 530 mil casos, com 256 mil óbitos mundialmente. No Brasil, este é o terceiro tipo mais prevalente e a quarta maior causa de mortalidade em mulheres, sendo o risco estimado de 17,11 casos a cada 100 mil mulheres (Barcerlos et al. 2017; Silva et al. 2020).

O câncer de colo uterino pode ser ocasionado por uma infecção persistente de diferentes tipos de Papilomavírus Humano (HPV) (INCA 2016). Este vírus tem a capacidade de causar lesões na pele e mucosas, sendo essas infecções frequentes e quase sempre regridem naturalmente. Além disso, existem mais de cem tipos de HPV, que podem ser classificados como de baixo ou alto risco oncogênico. Dos quarenta tipos que contaminam o trato ano-genital, o 16 e o 18 são os que mais prevalecem nos casos de câncer cervical invasivo, compreendendo uma porcentagem de $70 \%$ do total dessas neoplasias (Silva; Mendonça \& Perinazzo 2020; Libera et al. 2016).

As lesões precursoras podem ser detectadas através dos programas de rastreamento ou screening das mulheres, que acontecem através da realização do exame citopatológico, conhecido também como o exame de Papanicolau, que é uma maneira segura, efetiva e de baixo custo para o diagnóstico precoce de CCU antes mesmo do aparecimento dos sintomas (Garcia et al. 2021).

Durante as consultas ginecológicas, as vaginoses bacterianas (VB) e vulvovaginites representam a maioria das queixas ginecológicas. As vaginoses são processos em que o ambiente fisiológico do trato reprodutivo feminino está alterado, sendo 
associados a presença de inflamação ou não. Nos achados clínicos, as mulheres relatam a presença de corrimento vaginal, odor desagradável associado a coloração em diferentes aspectos, prurido, sensação de ardor e/ou queimação. Das espécies mais frequentemente encontradas em casos de VB são Gardnerella vaginallis, Clostridium ssp e Mycoplasmas, além dos achados na candidíase vaginal por Candida albicans, sendo este o agente mais frequente no processo inflamatório vaginal. Já em infecções de transmissão sexual tem como agente etiológico principalmente, o parasita flagelado Trichomonas vaginalis (Linhares et al. 2018; Ledger \& Witkin 2016).

A periodicidade na realização do exame citopatológico preconizada pelo Ministério da Saúde, assim como as estabelecidas pelos programas internacionais de controle do câncer, indicam que a faixa etária prioritária para realização do exame citopatológico é entre 25 a 60 anos de idade e sua realização deve ser uma vez ao ano e, depois de dois exames consecutivos com resultados negativos, deve-se realizar a cada três anos. Além de toda mulher que já teve sua primeira relação sexual antes dos 25 anos deve realizar o exame até completar os 60 anos de idade (Barbosa et al. 2017).

Assim, o objetivo do trabalho é analisar o perfil microbiológico e as alterações citológicas associadas as amostras cérvico-vaginais de mulheres atendidas em uma instituição filantrópica, no estado de Sergipe.

\section{Metodologia}

Trata-se de um estudo retrospectivo, descritivo e transversal. Com levantamento de dados, utilizando os prontuários das mulheres que realizaram o exame citopatológico entre os meses de julho à outubro de 2017 na instituição filantrópica: Legião Feminina de Educação e Combate ao Câncer (LFECC), localizada no estado de Sergipe.

Os dados coletados seguiram os seguintes critérios de inclusão: mulheres que realizaram o exame citopatológico no período de estudo, que não estavam fazendo uso de cremes vaginais ou antibióticos, todas as mulheres sexualmente ativas e que possuíam informações completas nos prontuários; os critérios de exclusão das participantes foram: mulheres virgens, pois, realizam coleta com o $s w a b$, apenas para pesquisa da microbiota, mulheres que estavam em uso de antibiótico e as que possuíam ausência de informações nos prontuários.

A coleta no banco de dados da instituição foi correspondente aos diagnósticos e dados epidemiológicos como: faixa etária das mulheres, adequabilidade das amostras cérvico-vaginais, presença de agentes infecciosos e achados citológicos.

A análise estatística do estudo foi realizada através do programa estatístico Graphpad Pad Prism 7.04 e o nível de significância adotado foi de $5 \%$.

Este estudo foi aprovado pelo Comitê de Ética em Pesquisa Envolvendo Seres da Universidade Tiradentes (CEP/UNIT) sob o número CAAE 68977217.7.0000.5371.

\section{Resultados e Discussão}

De julho a outubro de 2017, foram realizados cerca de 500 exames citopatológicos em mulheres atendidas na LFECC. Os resultados das amostras foram analisados quanto à sua adequabilidade, sendo diferenciados em satisfatórias ou insatisfatórias e observou-se que 96\% (480/500) das amostras foram consideradas satisfatórias e 4\% (20/500) insatisfatórias. As amostras insatisfatórias foram divididas em Insatisfatória por Sobreposição Celular representando 2\% (10/500) dos casos, Insatisfatória por Presença de Piócito em 1,2\% (6/500) e Insatisfatória por Sangue em 0,8\% (4/500), conforme a Gráfico 1. 
Gráfico 1- Adequabilidade das amostras cérvico-vaginais coletadas em mulheres atendidas na LFECC, no período de julho a outubro de 2017.

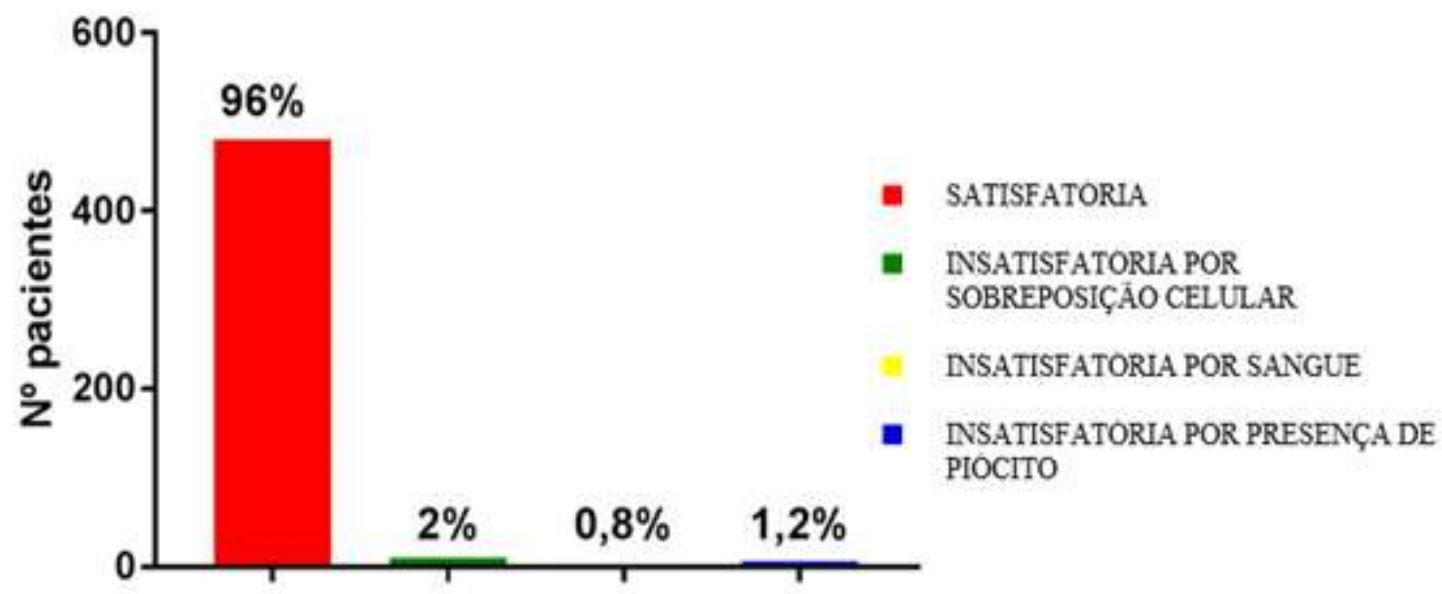

Fonte: Autores.

O Sistema Bethesda é utilizado na comparação dos resultados nacionais com aqueles encontrados nas publicações científicas internacionais. Este define que a adequabilidade da amostra pode ser satisfatória ou insatisfatória. Para o esfregaço ser considerado como satisfatório, é necessário a presença da Junção Escamo Colunar (JEC), ou seja, células epiteliais escamosas e glandulares. Porém, a presença de células glandulares não é considerada como critério incontornável para a leitura, mas a sua ausência deve ser relatada. Além disso, este sistema recomenda que a quantidade de células necessária para analisar possíveis alterações seja de até 75 células por campo, ou na maioria dos campos do microscópio com a objetiva de 4x, senão a amostra poderá ser considerada como insatisfatória (Brasil, 2006; Gamboni \& Miziara 2013).

Outros critérios para considerar as amostras insatisfatórias são a presença de mais de 75\% do esfregaço com sangue, infiltrado leucocitário, dessecamento, artefatos de estiramento, citólise e contaminação externa, estes podem prejudicar a leitura da amostra e interpretação dos resultados (Consolaro \& Maria-Engler 2016).

No presente estudo foi observado uma maior prevalência de amostras satisfatórias (96\%) em relação as amostras insatisfatórias (4\%), diferentemente do estudo realizado por Reis, em 2013, que as amostras satisfatórias correspondiam a $100 \%$ das amostras analisadas. Outro achado deste estudo, foram que as amostras Insatisfatórias por Sobreposição Celular foram superiores, com 2\%, em relação as Insatisfatórias por presença de Piócitos, com 1,2\% e as Insatisfatórias por Sangue, com $0,8 \%$. Os critérios de insatisfação verificados nas amostras podem ser decorrentes principalmente da má coleta, processos inflamatórios e/ou JEC ectópica.

Ao analisar o perfil microbiológico das amostras, nota-se as seguintes distribuições: Microbiota Mista em 31,2\% (156/500) dos casos, Cocos em 29,45 (147/500), Lactobacilos sem citólise em 16\% (80/500), Lactobacilos com citólise em 9,2\% (46/500), Bacilos supracitoplasmáticos (sugestivo de Gardnerella vaginalis/mobiluncus) em 7,4\% (37/500), Cocobacilos em 1,6\% (8/500), Cândida spp. em 2,4\% (12/500), Trichomonas vaginalis em 0,8\% (4/500) e ausência de microorganismos em $1,6 \%(8 / 500)$, segundo a Gráfico 2. 
Gráfico 2- Perfil microbiológico dos resultados encontrados em amostras cérvico-vaginais de mulheres atendidas na LFECC, no período de julho a outubro de 2017.

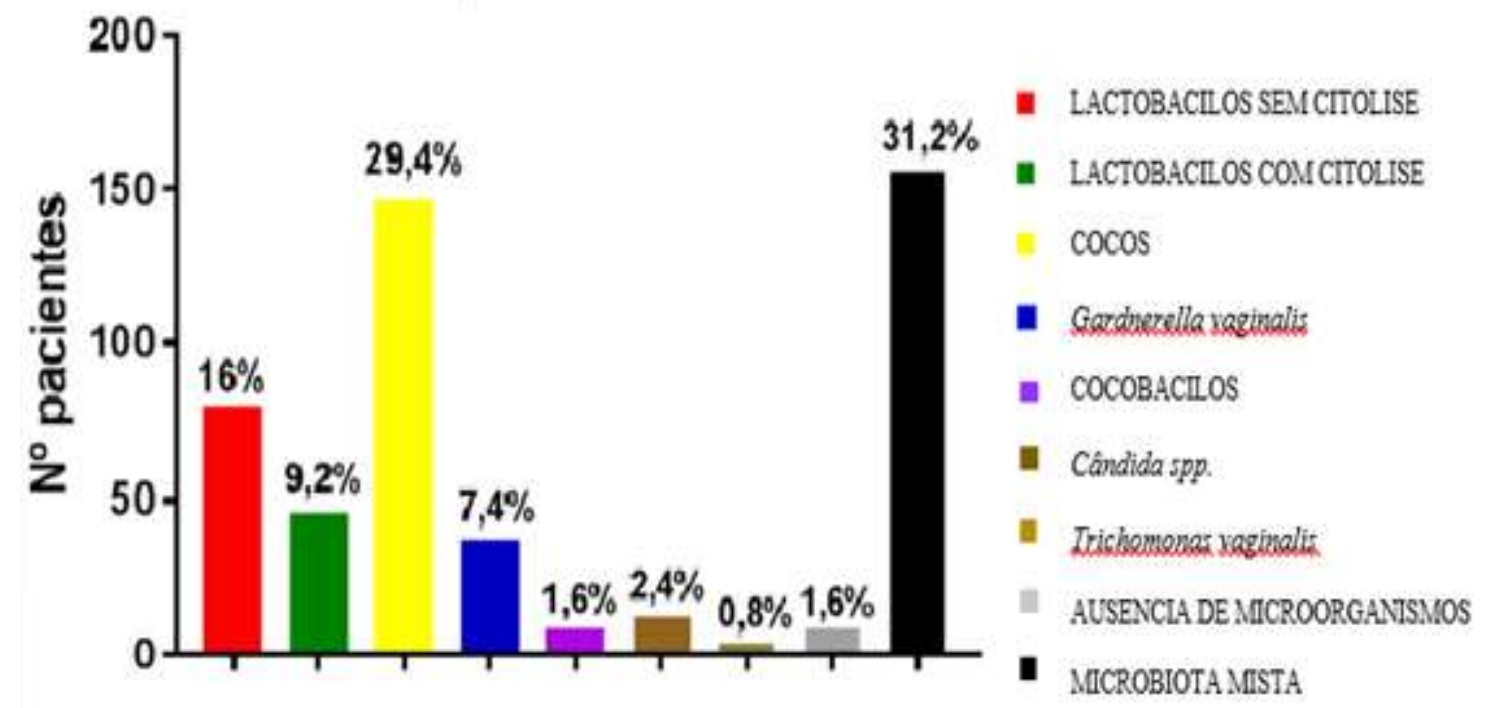

Fonte: Autores.

De acordo com os resultados analisados a microbiota mista (31,2\%), apresentou uma diferença estatística em relação a presença de outros microrganismos isolados, isso porque a vagina e o colo são formados por um complexo de inúmeras bactérias, de diferentes espécies, que podem ser, em circunstâncias exclusivas, a causa de cervicovaginites acompanhadas de corrimentos. Para que existam cervicovaginites, devem ser encontradas uma série de alterações gerais e citológicas nos esfregaços (Consolaro \& Maria-Engler 2012).

A microbiota cocoide é decorrente de locais onde o epitélio escamoso é pobre em glicogênio, ao contrário dos lactobacilos que estão presentes nas células do epitélio escamoso ricas em glicogênio no citoplasma. Os lactobacilos convertem o glicogênio em glicose e depois em ácido láctico que é responsável pela acidificação do pH vaginal, e é normal encontrar uma grande proliferação destes em mulheres grávidas e na fase lútea do ciclo menstrual, devido ao aumento de glicogênio nessas situações. Esse microrganismo pode apesentar-se sem citólise ou com citólise, a qual corresponde ao rompimento da membrana citoplasmática das células do epitélio escamoso (Soares; Baptista \& Tavares 2017).

Ao analisar os resultados verificou-se que os microrganismos apresentaram uma elevada prevalência: a Gardnerella vaginalis/mobiluncus (7,4\%), Cândida spp. (2,4\%) e Trichomonas vaginalis (0,8\%). A presença destes microrganismos em amostras ginecológicas é relatada por muitos autores, pois ocasionam inflamação na maioria das mulheres que realizam exames citopatológicos. O diagnóstico das inflamações e dos agentes microbiológicos é de extrema importância, principalmente porque a taxa de mulheres infectadas por algum tipo de microrganismo é elevada, fazendo com que estas procurem atendimentos ginecológicos devido a diferentes infecções e inflamações vaginais (Lessa 2012; Cardona 2012).

A identificação dos microrganismos que ocasionam inflamação e lesão no colo uterino é necessário pois estes podem estimular a substituição da mucosa do tipo glandular em mucosa recoberta por epitélio escamoso. Essa substituição é conhecida como metaplasia escamosa. E assim, devido ao processo de diferenciação celular exista uma maior propensão à replicação do Papilomavírus Humano (HPV), devido sua quantidade de DNA, favorecendo à disseminação do câncer do colo uterino (Anjos et al. 2010).

Quanto aos achados citológicos encontrados nas amostras cérvico-vaginais de mulheres atendidas na LFECC, os seguintes resultados foram encontrados: Inflamação em 73,6\% (368/500) dos casos, Dupla Alteração, ou seja, Atrofia com 
Inflamação e Metaplasia Escamosa Imatura e/ou Inflamação com Metaplasia Escamosa imatura 13,4\% (64/500) dos casos, Atrofia com Inflamação em 9,4\% (47/500), dentro dos limites da Normalidade em 3,4\% (17/500) e Metaplasia Escamosa Imatura em 1\% (5/500) dos resultados (Gráfico 3).

Gráfico 3- Achados citológicos dos resultados encontrados em amostras cérvico-vaginais em mulheres atendidas na LFECC, no período de julho a outubro de 2017.

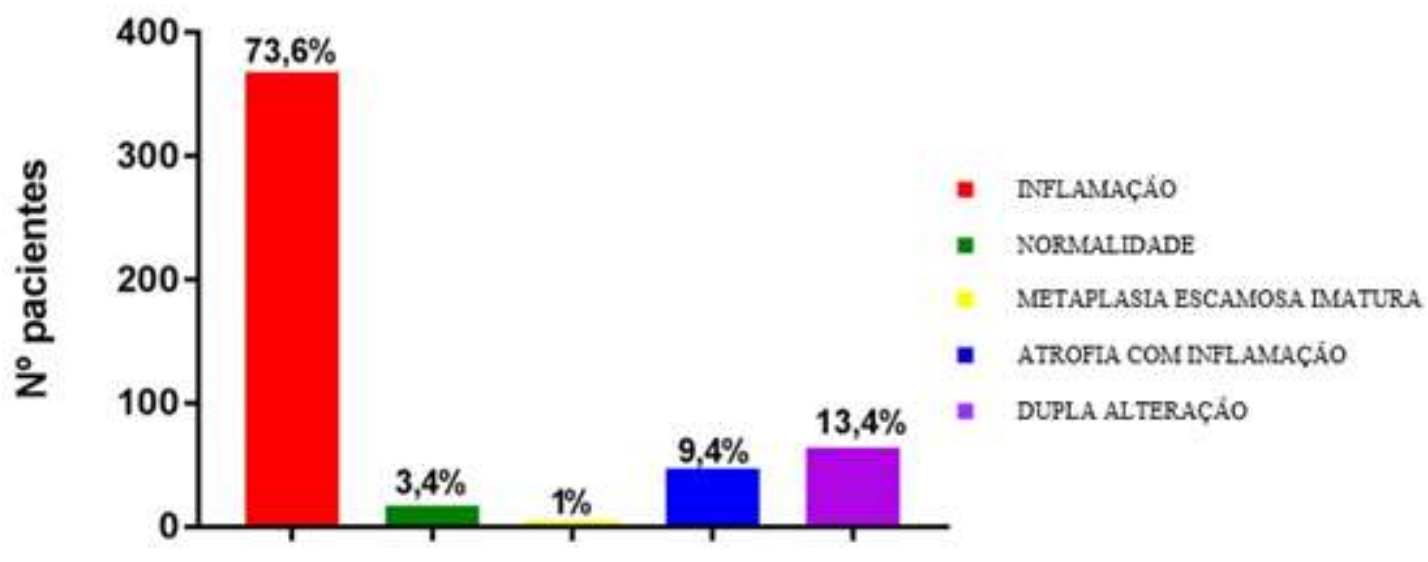

Fonte: Autores.

A grande maioria dos resultados citológicos analisados, as mulheres apresentaram inflamação, com 73,6\% dos casos, sendo que as amostras dentro dos limites da normalidade foram apenas 3,4\% dos resultados. A inflamação ou processo inflamatório é uma resposta complexa que se desenvolve devido aos agentes agressores teciduais. Podem ocorrer modificações de estruturas dos epitélios, como: hiperplasia, metaplasia, fenômenos de reparação e alterações morfológicas variadas, algumas comuns a todas as inflamações, outras representando modificações específicas do agente e, na citologia, às vezes, é possível detectar o agente causal (Araújo; Santana \& Arisawa 2013).

Além da inflamação, o epitélio escamoso pode apresentar-se metaplásico, ou seja, quando uma célula madura (epitelial ou do mesênquima) é substituída por outra de outro tipo celular. A metaplasia escamosa imatura, que apresentou apenas $1 \%$ dos resultados, caracteriza-se no esfregaço por serem do tamanho das células parabasais ou intermediárias, citoplasma abundante, cianófilo, núcleo aumentado, binucleação e nucléolos evidentes. Um esfregaço atrófico apresenta-se com um grande número de células parabasais e basais, principalmente em mulheres menopausadas.

\section{Conclusão}

Portanto, o exame citopatológico é uma ferramenta essencial para o rastreamento e prevenção do câncer do colo uterino, além da identificação de lesões citológicas e microorganismos percussores do CCU.

Ao avaliar a adequabilidade das amostras cérvico-vaginais, a maioria apresentou um resultado positivo, ou seja, amostras com adequabilidade satisfatórias. Porém, um elevado número de microorganismos encontrados nas mulheres foram relativamente elevados, principalmente de Gardnerella vaginalis. Já em relação as alterações citológicas, foi observado que a inflamação estava presente na maioria dos casos, seguida da normalidade.

Assim, observou-se que os resultados apresentados são relevantes, pois a distribuição dos números de casos de microorganismos e alterações citológicas foram elevados. Com isso, demonstra-se a importância da realização de pesquisas futuras para acompanhar os achados nos exames citológicos em mulheres, com o objetivo de detectar as alterações citológicas e microorganismos percussores do CCU e assim contribuir para melhorias na saúde da população feminina. 


\section{Referências}

Anjos, S. J. S. B. et al. (2010). Fatores de risco para câncer do colo do útero segundo resultados de IVA, citologia e cervicografia. Rev. Esc. Enferm. 44 (4), 912-920.

Araújo, E. C. C., Santana, R. J. \& Arisawa, E. A. S. (2013). Mecanismos da inflamação: análise dos processos fisiopatológicos. < http://www.inicepg.univap.br/cd/INIC_2013/anais/arquivos/0839_0637_01.pdf >.

Barcelos, M. R. B. et al. (2017). Qualidade do rastreamento do câncer de colo uterino no Brasil: avaliação externa do PMAQ. Revista de Saúde Pública, 51, 113.

Barbosa, L. C. R. et al. (2017). Percepção de mulheres sobre os fatores associados a não realização do exame papanicolau. Interfaces Científicas-Saúde e Ambiente, 5 (3), 87-96.

Brasil. (2006). Ministério da Saúde. Secretaria de Atenção à Saúde. Instituto Nacional de Câncer. Coordenação de Prevenção e Vigilância. Nomenclatura brasileira para laudos cervicais e condutas preconizadas: recomendações para profissionais de saúde. INCA. <http: //bvsms.saude.gov.br/bvs/publicacoes/Nomenclaturas_2_1705.pdf>.

Cardona, Y. T. et al. (2012). Prevalencia de citologia anormal e inflamación y suasociaciónconfactores de riesgo para neoplasias delcuello uterino enelCauca, Colombia. Rev. Salud Pública, 14 (1), 53-66.

Consolaro, M. E. L \& Maria-Engler, S. S. (2016). Citologia clínica cérvico-vaginal-texto e atlas. Roca.

Consolaro, M. E. L \& Maria-Engler, S. (2012). Citologia clínica cérvico-vaginal-texto e atlas. Roca.

Gamboni, M. \& Miziara, F. E. (2013). Manual de citopatologia diagnóstica. Manole,1ed.

Garcia, M. et al. (2021). Identificação dos fatores que interferem na baixa cobertura do rastreio do câncer de colo uterino através das representações sociais de usuárias dos serviços públicos. Brazilian Journal of Health Review, 4 (1), 1462-1477.

INCA.Instituto Nacional de Câncer. José Alencar Gomes da Silva. (2016). <http://www2.inca.gov.br/wps/wcm/connect/tiposdecancer/site/ho me/colo_ut er o/definicao>.

Lessa, P. R. M. et al. (2012). Presença de lesões intraepiteliais de alto grau entre mulheres privadas de liberdade: Estudo documental. Rev. Latino-Am. Enfermagem, 20 (2), 354-361.

Ledger, W. J. \& Witkin, S. S. (2016). Vulvovaginal infections. 2nd ed. Boca Raton (FL): CRC Press Taylor \& Francis Group, Chapter 2, Vaginal immunology. 7-12.

Libera, L.S.D. et al. (2016). Avaliação da infecção pelo Papiloma Vírus Humano (HPV) em exames citopatológicos. RBAC, 48 (2), $138-43$.

Linhares, I. M. et al. (2018). Vaginites e vaginoses. São Paulo: Federação Brasileira das Associações de Ginecologia e Obstetrícia (Febrasgo). (Protocolo Febrasgo - Ginecologia, $n^{\circ}$ 24/Comissão Nacional Especializada em Doenças Infectocontagiosas).

Reis, N. R. O. G. et al. (2013). Perfil microbiológico e alterações citológicas associadas em material cérvico-vaginal coletado em consultório de enfermagem, de 2009 a 2011 em Aracaju/SE. ScientiaPlena, 9 (5), 1-8.

Soares, R., Baptista, P. V. \& Tavares, S. (2017). Vaginose citolítica: uma entidade subdiagnosticada que mimetiza a candidíase vaginal. Acta Obstétrica e Ginecológica Portuguesa, 11 (2), 106-112.

Silva, M. L. et al. (2020). Conhecimento de mulheres sobre câncer de colo do útero: Uma revisão integrativa. Brazilian Journal of Health Review, 3 (4), 7263 7275 .

Silva, G. C. M. C., Mendonça, M. C. \& Perinazzo, V. M. (2020). Tipos histológicos do câncer do colo do útero associado com a infecção pelo HPV em pacientes atendidas em hospital de referência oncológica no estado do Pará. Revista Eletrônica Acervo Científico, 14, 1-8.

Taquary, L. R. et al. (2018). Fatores de risco associados ao Papiloma vírus Humano (HPV) e o desenvolvimento de lesões carcinogênicas no colo do útero: uma breve revisão. CIPEEX, 2, 855-859. 